\title{
¿Movilidad social a partir de la movilidad geográfica?: los casos de familias argentinas inmigradas en un contexto regional español
}

\author{
Sofía Laíz Moreira \\ Laboratoire Méditerranéen de Sociologie (LAMES). \\ CNRS - Aix-Marseille Université / Enseignante \\ au Département d'Études Hispaniques et Latino- \\ américaines (DEHLAM) / ALLSH - Aix-Marseille \\ Université , Argentina
}

Recepción: 20 Enero 2019

Aprobación: 01 Junio 2020

Publicación: 02 Agosto 2020

Cita sugerida: Laíz Moreira, S. (2020). ¿Movilidad social a partir de la movilidad geográfica?: los casos de familias argentinas inmigradas en un contexto regional español. Cuestiones de Sociología, 23, e105. https:// doi.org/10.24215/23468904e105
Resumen: Este artículo presenta los resultados de una investigación cualitativa realizada entre los años 2011 y 2015 y cuyo objetivo principal fue el de estudiar los procesos de movilidad social intergeneracional de 10 grupos familiares de migrantes argentinos asentados en la Comunidad Autónoma de Galicia, España. La metodología empleada respondió a un estudio de tipo etnográfico y multi-situado, entrevistando no solo a los miembros de las diferentes generaciones de las familias objeto de estudio sino también a otros miembros de las comunidades de origen de los migrantes, así como a informantes claves que contribuyeron a explicar las características de los contextos sociales de origen en términos de sus estructuras educativa y ocupacional. De esta forma, se pudo acceder a informaciones útiles para así poder situar las posiciones sociales y educativas ocupadas por las generaciones de padres en la situación previa al proceso migratorio y postmigración. Los resultados de tales mediciones del nivel educativo entre generaciones, y de las categorías ocupacionales entre los espacios sociales involucrados, dejaron ver unos movimientos que reflejan y confirman las teorías acerca de la influencia del origen social sobre las trayectorias educativas de los hijos, pero que también ponen de relieve el importante impacto de los factores macroestructurales, tales como las políticas educativas, las características de la estructura laboral de la sociedad de instalación, la situación coyuntural de la economía local al momento del estudio y los procesos de discriminación por origen étnico dentro de la sociedad española.

Palabras clave: Migraciones, Movilidad social intergeneracional, Trayectorias educativas, Argentina, España, Galicia.

Abstract: This paper presents the results of a qualitative research work carried out between 2011 and 2015 and which main objective was to study the intergenerational social mobility strategies of 10 family groups of two concrete origins (Argentinean and Moroccan) settled in the Autonomous Community of Galicia, in Spain. The methodology used has included an ethnographic and multi-sited study, conducting interviews to different family members of different family generations as well as to other family members still living in the country of origin and key informants that's helped to understand the characteristics of educational and professional structures in pre-migration social contexts. The results of these measurements in regard to the educational attainment and 
professional categories between generations has confirmed the impact of social origin in descendants' pathways but also the influence of the structural determinants, as is the education policy, the characteristics of the occupational structure in the immigration context, the socio-economic situation at the moment of this study and the discrimination processes in the Spanish society.

Keywords: International migrations, Intergenerational social mobility, Capitals, Argentina, Spain, Galicia.

\section{INTRODUCCIÓN}

Las migraciones argentinas hacia España se caracterizan por mostrar unas tendencias que dividen históricamente a sus flujos de emigración en diferentes corrientes que coinciden con cinco fases cíclicas marcadas por momentos de crisis política, social o económica en este país austral. Estas fases se corresponden con una primera oleada que se inicia en los años 70 , conocida como de "fuga de cerebros", la del exilio político que comienza con el golpe de estado de 1976, la de la hiperinflación del año 1989, la de la etapa neoliberal desde 1997 al año 2000 y la que comienza con la crisis del 2001, también conocida como la crisis del "corralito" (Actis y Esteban, 2007).

Este texto presenta un análisis de un conjunto de historias familiares de argentinos que salieron de su país hacia la Comunidad Autónoma de Galicia, en España, fundamentalmente en la última de las etapas mencionadas, aunque también se incluyeron casos de otras fases migratorias, en particular de la hiperinflacionaria y neoliberal. El objetivo de este análisis es el de responder a la pregunta de qué movimientos se produjeron entre las generaciones de padres e hijos desde las dimensiones educativa y ocupacional. De esta forma, el estudio de los procesos de movilidad social intergeneracional a partir de la movilidad geográfica de estas familias aplicó una perspectiva longitudinal, para luego medir las diferencias de los niveles educativos y de la categoría profesional alcanzada por el o la joven tomado como punto de medición entre la generación de los descendientes.

Para ello, el análisis se desarrolló desde una perspectiva histórica de cada grupo filial, incluyendo, en ocasiones, a los abuelos de los jóvenes estudiados; aunque estos solo fueron reflejados en las historias grupales, pues no fueron considerados objeto del análisis de los movimientos entre categorías (ocupacionales y educativas). Los casos fueron, así, clasificados en tres tipos de movilidad educativa/ocupacional: la "movilidad ascendente", la "movilidad descendente" y la "reproducción del nivel educativo/ocupacional". Es importante remarcar que, cuando se procedió a la clasificación de los casos, la comparativa intergeneracional tuvo en cuenta a ambos progenitores, tanto a la madre como al padre, en un afán por mostrar "la situación real (diversa y con matices) rompiendo con el supuesto sexista de asignar a toda la unidad familiar la posición de clase del cabeza de familia" (Requena, Salazar y Radl 2012, p. 91). Thélot (1991), en los años 90, ya explicaba cómo el nivel de formación de la madre comenzaba a ganar en importancia, siendo añadida a la posición del padre. Las investigaciones sobre movilidad social en España, de hecho, corroboran esta premisa pues revelan una fuerte correlación entre el nivel de estudios de la madre y los resultados académicos de los descendientes en el ámbito escolar (Feito Alonso, 2010, p. 50).

En el siguiente apartado, definiremos los conceptos básicos que servirán en adelante para establecer los patrones de movilidad educativa y ocupacional de las familias estudiadas. 


\section{MARCo metodológico}

Este trabajo se basó en un estudio de tipo cualitativo en base a entrevistas en profundidad e historias de vida realizadas a 10 grupos familiares. Las familias fueron localizadas a partir de la selección de jóvenes migrantes respondiendo a los perfiles de estudio definidos para esta investigación: jóvenes de segunda generación y de generación $1.5^{2}$ de familias argentinas residiendo en la Comunidad Autónoma de Galicia.

La localización de los informantes fue realizada a partir de la técnica de "bola de nieve" a partir del contacto con entidades asociativas. Se ha tenido, para ello, consideración de un equilibro respecto del género y de la ciudad de instalación de las familias, siendo a partes iguales distribuidas entre las ciudades de Vigo y A Coruña, polos de mayor acogida de flujos migratorios en esta región. Para completar el abordaje del análisis de movilidad social a partir de la movilidad geográfica, la comprensión de la distribución de categorías educativas y ocupacionales en la sociedad de origen de los migrantes fue completada con entrevistas a informantes claves (miembros del sistema educativo, representantes de la autoridad educativa local, miembros de las comunidades locales de origen de los migrantes: vecinos, amigos, miembros de la familia extensa). De esta forma, se buscó la comprensión de la estructura social de procedencia para poder realizar un análisis más adecuado del cambio de las categorías socioprofesionales previas y posteriores a la migración y entre sistemas sociales diferenciados.

Pasaremos a continuación a presentar el esquema clasificatorio mediante el cual se ha realizado la medición de los movimientos en el ámbito ocupacional y que complementarán el análisis sobre la movilidad educativa entre generaciones:

CUADRO 1

Clasificación Socioeconómica Europea (ESeC)

\begin{tabular}{|l|}
\hline 1 Grandes empleadores, directivos y profesionales de nivel alto \\
\hline 2 Directivos y profesionales de nivel bajo \\
\hline 3 Empleados de cuello blanco de nivel alto \\
\hline 4 Pequeños empleadores y trabajadores autónomos no agrícolas \\
\hline 5 Trabajadores autónomos agrícolas \\
\hline 6 Supervisores y técnicos de rango inferior \\
\hline 7 Trabajadores de los servicios y comercio de rango inferior \\
\hline 8 Trabajadores manuales cualificados \\
\hline 9 Trabajadores no cualificados \\
\hline 10 Excluidos del mercado de trabajo y parados de larga duración \\
\hline
\end{tabular}

Fuente: Informe CECS Fundacion Encuentro 2011.

La escala presentada corresponde a la Clasificación Socioeconómica Europea (ESeC). ${ }^{3}$ La misma presenta diez categorías ocupacionales, entre las cuales encontramos los excluidos del mercado de trabajo y los parados de larga duración. En este punto reside el principal interés de incluir este esquema clasificatorio y no otro. En cada categoría se integran los niveles educativos requeridos normalmente para el desarrollo del empleo en la naturaleza de cada ocupación, y se trata de la escala empleada a nivel europeo y que utiliza el propio Instituto Nacional de Estadística en España. El esquema proporcionado por el International Standard Classification of Occupations (CIUO-88 -en español- o ISCO-88) es la escala de estratificación anterior al 
esquema ESeC. Sin embargo, esta no tiene integrados los niveles educativos en cada categoría ocupacional pues propone la combinación entre ambas variables, lo que puede producir un sesgo frente al fenómeno de la sobrecualificación o de la subocupación, por ejemplo. Pese a ello, lo que nos interesa cuando hablamos de movimientos en la escala social es la categoría socioprofesional alcanzada dentro del mercado laboral, pues el nivel educativo no garantiza la inserción en la categoría profesional esperada; en otras palabras, se puede contar con un título de formación superior y pese a ello no ocupar un puesto acorde a este nivel de formación. Por otra parte, este sesgo es compensado por la posibilidad que ofrece la escala seleccionada de medir los casos de "excluidos del mercado de trabajo y parados de larga duración", lo que nos permitirá medir la movilidad en los casos de miembros en situación de desempleo. De esta manera, los jóvenes o padres en situación de desocupación se consideraran dentro de la categoría 10 (excluidos del mercado de trabajo y parados de larga duración), si bien reconocemos que se puede tratar de una situación temporal.

Este estudio fue realizado entre los años 2011-2015. Parte de él fue financiado por la Agencia española de cooperación al desarrollo del Gobierno de España y la Oficina de cooperación y voluntariado de la Universidade da Coruña (Galicia).

\section{MARCo TEÓRICo}

En Marx y Weber encontramos el enfoque más clásico sobre la estratificación social. A grandes rasgos, las diferencias entre ambas posturas se resumen en que, mientras Marx concibe la sociedad como una lucha de clases y establece la explotación como principio de las relaciones de producción, Weber da lugar a la acción social como mecanismo de cambio, diferenciando la clase social del status. Para Weber, la primera sería definida como principio de estratificación económica, mientras que el segundo se definiría como criterio de estratificación según la estimación social. Será luego Goldthorpe quien tome el concepto de status social, o más bien, como lo denomina, de "deseabilidad social de las ocupacionales" para diseñar la escala de categorías ocupacionales, basada en su concepción sobre la categorización empírica de clases sociales (Goldthorpe y Hope, 1974). He aquí la semilla de lo que otros autores denominarán el "status subjetivo" como "una forma de autopercepción de su status social y que se asocia a sentimientos de inferioridad o superioridad social" (Ichou, 2018, p. 175). Existen diversas corrientes teóricas que abordan el tema de la estratificación y de la movilidad social en las sociedades modernas. Entre ellas, empero, solo tendremos en cuenta la teoría de la industrialización, las teorías de la reproducción social y las teorías sobre la proletarización.

Fueron los planteamientos funcionalistas de los años cincuenta y sesenta los que impulsaron los estudios sobre movilidad social en los Estados Unidos desde la teoría de la industrialización. Desde esta teoría, los teóricos liberal-funcionalistas sostienen que la institución educativa pasa a ocupar el papel preponderante en la movilidad social, en función de la igualdad de oportunidades y el logro diferencial (Echeverría Zabalza, 1999, p. 95). Por el contrario, la corriente marxista niega que se produzca mayor igualdad, mayor apertura y mayor oportunidad de promoción social en las sociedades industriales. De esta forma, los seguidores del enfoque más clásico rechazarán la tesis funcionalista a favor de la idea de una reproducción de la clase social, donde el patrón repetido será la inamovilidad. Sobre ello sentará su base la teoría de la reproducción social y de la correspondencia. Si la noción de reproducción social se basa en la idea de una transmisión de la posición social de padres a hijos, la noción de correspondencia de Bowles y Gintis (1976) contendrá la idea de que existe una correspondencia entre las relaciones sociales que tienen lugar en la familia y en la escuela con aquellas que mantiene el cabeza de familia en el ámbito productivo. En tajante oposición a la perspectiva funcionalista, los autores marxistas sostienen que lo que tiene lugar es la reproducción casi inevitable de la clase social de los padres a los hijos (Echeverría Zabalza, 1999, p. 96) y que la institución escolar beneficia a los grupos con mayor capital humano y mejor posición socioeconómica (Bourdieu,1979; Feito Alonso, 2010, p. 49). Así también lo constatan otros trabajos sobre movilidad social y migración en Europa (Ichou, 2015 y 2018; Laiz, 2016), en recientes estudios aplicados a los hijos de inmigrantes en Francia, concluyendo 
que las trayectorias escolares de los hijos de inmigrantes están principalmente sujetas al origen social ${ }^{4}$ y a la categoría social previa y posterior a la migración familiar. Este abordaje, lógicamente, tiene sus bases en el teórico pionero de la reproducción social, Pierre Bourdieu. Su trabajo junto a Passeron (Bourdieu y Passeron, 1970) ha introducido el concepto de reproducción familiar, reconociendo la reproducción de las jerarquías sociales en la escuela. De esta forma, las desigualdades de oportunidades en el ámbito escolar se reconocen condicionadas por el origen familiar.

Por último, las teorías de la proletarización, encabezadas por Poulantzas (1973), establecen que en las sociedades modernas existe una tendencia hacia la movilidad descendente y hacia la descualificación, aportando la visión más pesimista a nuestro debate.

En el caso de este análisis, y con el objetivo de estudiar la movilidad social de los migrantes a partir de un anclaje intergeneracional, tomando la trayectoria educativa de los hijos pero también los procesos de inserción de los padres, hemos tomado como marco teórico de referencia las teorías de Portes y Böröcz (1989) sobre los modos de incorporación al mercado de trabajo. Estos autores sostienen que dichos mecanismos se encuentran segmentados según el nivel ocupacional de origen, para lo cual establecieron una clasificación basada en tres categorías. La primera hace referencia a los menos afortunados en el proceso de incorporación a la sociedad de instalación (los "incapacitados" o "handicapped"). Estos se incorporarían de manera diferenciada dependiendo de la categoría ocupacional alcanzada en origen. Así, mientras aquellos cuyo origen ocupacional de tipo manual se posicionarían en el mercado secundario o sumergido, aquellos cuyo origen ocupacional corresponde a un puesto de categoría profesional se posicionarían dentro del mercado étnico, es decir, en tanto proveedores de servicios a la comunidad de origen asentada en el contexto de inmigración (Portes y Böröcz 1989, p. 620).

Una segunda categoría posicionaría a aquellos migrantes denominados "neutrales", ubicados dentro del mercado mixto si fueran de un origen ocupacional manual, es decir, un mercado compuesto tanto por proveedores étnicos así como proveedores del mercado local. Si fueran de origen profesional, se instaurarían en el sector del mercado primario, y si fueran de origen empresarial, acabarían insertándose como parte de la clase media comerciante local.

Aquellos más aventajados si bien contando con un nivel ocupacional de origen manual, mostrarían un proceso de incorporación relacionado con una movilidad social ascendente dentro del sector empresarial local, siendo incorporados a la clase media o "mainstream". Por su parte, aquellos de origen profesional lograrían insertarse en capas más altas de la sociedad, produciendo también una movilidad social ascendente hacia puestos de liderazgo, y aquellos de origen empresarial se incorporarían como empresarios dentro de la economía llamada de enclave (Portes y Böröcz, 1989, p. 620).

Finalmente, aquellos cuyo origen ocupacional coincide con un puesto de mando se posicionarían, una vez insertados en el nuevo contexto social, en lo que Blalock (1967) denominó "middle man minority", haciendo referencia a aquellos puestos que juegan un papel de intermediación entre diferentes actores sociales (entre el consumidor y el productor, entre el empleado y el empleador, entre el propietario y el arrendatario, entre las élites y las masas, etc.). En concreto, estos, los más aventajados, se ubicarían en puestos dentro del sector del comercio y las finanzas; ejemplos de ello son los agentes inmobiliarios o agentes colocadores de empleo, agentes de cambio, corredores de bolsa, etc. (Bonacich, 1973).

Ahora bien, para dar paso al análisis de las historias familiares, resulta imprescindible definir qué entendemos por movilidad social y por movilidad social intergeneracional. Para ello, tomamos como referencia la definición que hace Echeverría Zabalza (1998) sobre la movilidad, descripta como:

el movimiento o paso de determinados individuos de unas categorías sociales a otras diferentes, o de una posición a otra dentro de una determinada escala social. (...) La movilidad intergeneracional, por su parte, quiere significar el cambio que tiene lugar entre la ocupación, sector, clase estrato, etc., del padre al hijo o los hijos cuando dicho cambio se produce (Echeverría Zabalza, 1998, p. 63) 
De esta forma, la movilidad laboral se refiere a cambios individuales de posiciones en una escala de categorías laborales, o bien a movimientos entre sectores de actividad económica o geográfica (Miguélez., 2011, p. 135), es decir, al ascenso, descenso o reproducción que se produce entre diferentes categorías ocupacionales (Echeverría Zabalza, 1998, p. 64). La movilidad educativa, por su parte, reflejaría el cambio entre categorías equivalentes a los diferentes niveles de cualificación y, desde el análisis cuantitativo, nos indicaría el grado de correlación entre el nivel de escolarización de los padres y de los hijos (Sánchez Hugalde, 2004, p. 3). Precisamos, más aún, explicar cómo entendemos los diferentes movimientos entre categorías para medir el tipo de movilidad educativa y/o ocupacional. De esta forma:

- la movilidad educativa ascendente se entendería como el proceso por el cual el sujeto de estudio muestra una mejora en el nivel formativo respecto de aquel que ostentan sus padres y descendente cuando el sujeto tomado de la generación de los hijos rebaja el nivel educativo obtenido por sus progenitores.

- la movilidad ocupacional ascendente, cuando el sujeto de estudio obtiene una categoría ocupacional mejorada en comparación a la de sus padres y descendente cuando el sujeto tomado de la generación de los hijos empeora la categoría ocupacional -es decir, obtiene un puesto de trabajo de menor categoría ocupacional-de sus padres.

- Por último, la reproducción del nivel educativo, cuando el nivel formativo se reproduce de padres a hijos, y reproducción del nivel ocupacional cuando la categoría ocupacional es la misma entre ambas generaciones.

En el apartado a continuación presentaremos el resultado de la medición de los movimientos entre escalas sociales, centrándonos en la diferencia del nivel educativo y ocupacional entre padres y el o la joven tomado/ a para la medición, en lo que respecta a la generación de los descendientes.Como ya hemos comentado en el apartado metodológico, no todos los casos estudiados (10 familias) han permitido extraer conclusiones sobre los procesos de incorporación al mercado de trabajo de los descendientes, ${ }^{5}$ y por ello solo se incluirán en los resultados las familias cuyos hijos han ya comenzado la trayectoria laboral (8 casos).

\section{REsultados DE MOVILIDAD SOCIAL INTERGENERACIONAL DESDE LAS DIMENSIONES EDUCATIVA Y OCUPACIONAL}

A partir de la descripción de las trayectorias familiares hemos podido extraer algunas conclusiones sobre patrones comunes de movilidad social, tomando las dimensiones educativa y ocupacional. Las historias familiares tomadas como casos ilustrativos de estos cambios en las categorías sociales de los individuos fueron analizadas a la luz de las teorías presentadas en el epígrafe metodológico.

Una de las características más relevantes delas historias migratorias familiares estudiadas resulta en el fuerte componente intergeneracional del proyecto migratorio, lo que permite observar la evolución histórica desde la generación de los abuelos hasta los nietos. La tendencia observada en estas familias es de un predominio de mejora del nivel educativo de padres a hijos, aunque este no se traduce siempre en una mejor posición dentro del mercado laboral. Vemos los resultados de las trayectorias tomadas para este artículo en el cuadro a continuación: 


\section{CUADRO 1A}

Cambios intergeneracionales en las categorías educativas y ocupacionales de los migrantes. Generaciones de padres e hijo/a objeto de medición.

\begin{tabular}{|c|c|c|c|c|c|}
\hline & \multirow[t]{2}{*}{$\begin{array}{l}\text { Sujeto } \\
\text { descendiente }\end{array}$} & \multicolumn{2}{|c|}{ CSP de los padres antes de migrar } & \multicolumn{2}{|c|}{$\begin{array}{l}\text { CSP de los padres tras la } \\
\text { migración }\end{array}$} \\
\hline & & Padre & Madre & Padre & Madre \\
\hline 1 & Federico & $\begin{array}{l}\text { Profesional autónomo, } \\
\text { empresario }\end{array}$ & $\begin{array}{l}\text { Gestora de } \\
\text { negocio } \\
\text { familiar, } \\
\text { empresaria }\end{array}$ & $\begin{array}{l}\text { Profesional } \\
\text { autónomo, } \\
\text { empresario }\end{array}$ & $\begin{array}{l}\text { Gestora de } \\
\text { negocio } \\
\text { familiar, } \\
\text { empresaria }\end{array}$ \\
\hline 2 & Tomás & $\begin{array}{l}\text { Chofer, empleado } \\
\text { asalariado sin } \\
\text { cualificación }\end{array}$ & $\begin{array}{l}\text { Ama de casa - } \\
\text { (parada de } \\
\text { larga duración) }\end{array}$ & $\begin{array}{l}\text { Chofer, } \\
\text { empleado } \\
\text { asalariado sin } \\
\text { cualificación }\end{array}$ & Desocupada \\
\hline 3 & Moira & $\begin{array}{l}\text { Chofer, empleado } \\
\text { asalariado sin } \\
\text { cualificación }\end{array}$ & $\begin{array}{l}\text { Empleada } \\
\text { doméstica }\end{array}$ & $\begin{array}{l}\text { Chofer, } \\
\text { empleado } \\
\text { asalariado sin } \\
\text { cualificación }\end{array}$ & camarera \\
\hline 4 & Francisco & $\begin{array}{l}\text { Empleado asalariado } \\
\text { del sector de la } \\
\text { construcción (sin } \\
\text { cualificación) }\end{array}$ & $\begin{array}{l}\text { Propietaria y } \\
\text { gestora de } \\
\text { pequeño } \\
\text { comercio }\end{array}$ & $\begin{array}{l}\text { Comerciante } \\
\text { autónomo }\end{array}$ & $\begin{array}{l}\text { Empleada } \\
\text { asalariada, } \\
\text { limpieza } \\
\text { industrial }\end{array}$ \\
\hline 5 & Catalina & $\begin{array}{l}\text { Técnico electrónico, } \\
\text { técnico mecánico, } \\
\text { comerciante autónomo }\end{array}$ & $\begin{array}{l}\text { Docente de } \\
\text { educación } \\
\text { infantil }\end{array}$ & $\begin{array}{l}\text { Empleado } \\
\text { asalariado, } \\
\text { comercial }\end{array}$ & $\begin{array}{l}\text { Empleada } \\
\text { asalariada, } \\
\text { comercial }\end{array}$ \\
\hline 6 & Marta & Artesano autónomo & $\begin{array}{l}\text { Artista plástica } \\
\text { independiente }\end{array}$ & $\begin{array}{l}\text { Artesano, } \\
\text { comerciante }\end{array}$ & $\begin{array}{l}\text { Artesana, } \\
\text { comerciante }\end{array}$ \\
\hline 7 & Martin & $\begin{array}{l}\text { Chofer, empleado } \\
\text { asalariado de baja } \\
\text { cualificación }\end{array}$ & No migrante & $\begin{array}{l}\text { Empleado } \\
\text { baja } \\
\text { cualificación, } \\
\text { sector } \\
\text { construcción }\end{array}$ & No migrante \\
\hline 8 & Nicolás & $\begin{array}{l}\text { Profesional cualificado } \\
\text { y pequeño empleador } \\
\text { (empresa familiar) }\end{array}$ & $\begin{array}{l}\text { Profesional } \\
\text { cualificada } \\
\text { independiente }\end{array}$ & $\begin{array}{l}\text { Empleada } \\
\text { cuello blanco, } \\
\text { sector oficinas } \\
\text { y despachos }\end{array}$ & $\begin{array}{l}\text { Empleada } \\
\text { cuello blanco, } \\
\text { sector } \\
\text { oficinas y } \\
\text { despachos }\end{array}$ \\
\hline
\end{tabular}


CUADRO 1B

Cambios intergeneracionales en las categorías educativas y ocupacionales de los migrantes. Generaciones de padres e hijo/a objeto de medición.

\begin{tabular}{|c|c|c|}
\hline \multicolumn{2}{|c|}{ Resultado de movilidad social } & \multirow[t]{2}{*}{ Descripción itinerario } \\
\hline Dimensión educativa & $\begin{array}{c}\text { Dimensión } \\
\text { ocupacional }\end{array}$ & \\
\hline $\begin{array}{l}\text { De padres con nivel de estudios } \\
\text { secundarios a hijo con nivel de estudios } \\
\text { universitarios }\end{array}$ & $\begin{array}{l}\text { Movilidad } \\
\text { ocupacional } \\
\text { ascendente }\end{array}$ & $\begin{array}{l}\text { De padre y madre con categoría } \\
\text { ocupacional } 4 \text { a hijo con categoría } \\
\text { ocupacional } 1\end{array}$ \\
\hline $\begin{array}{l}\text { De padres con nivel de estudios } \\
\text { secundarios sin terminar a hijo con nivel } \\
\text { de formación profesional de grado medio }\end{array}$ & $\begin{array}{l}\text { Movilidad } \\
\text { ocupacional } \\
\text { ascendente }\end{array}$ & $\begin{array}{l}\text { De padre con categoría ocupacional } 9 \text { y } \\
\text { madre con categoría ocupacional } 10 \text { a } \\
\text { hijo con categoría ocupacional } 7 \\
\text { (trabajador de los servicios y comercio } \\
\text { de rango inferior) }\end{array}$ \\
\hline $\begin{array}{l}\text { De padre con nivel de estudios } \\
\text { secundarios y madre con nivel de } \\
\text { estudios primarios a hija con nivel de } \\
\text { formación profesional }\end{array}$ & $\begin{array}{l}\text { Movilidad } \\
\text { ocupacional } \\
\text { descendente }\end{array}$ & $\begin{array}{l}\text { De padre con categoría } 9 \text { y madre } \\
\text { categoría } 9 \text { a hija en situación de } \\
\text { desempleo (categoría 10) }\end{array}$ \\
\hline $\begin{array}{l}\text { De padres con nivel de estudios } \\
\text { secundarios a hijo con nivel de } \\
\text { formación profesional }\end{array}$ & $\begin{array}{l}\text { Movilidad } \\
\text { ocupacional } \\
\text { descendente }\end{array}$ & $\begin{array}{l}\text { De padre categoría } 4 \text { sector servicios } \\
\text { (autoempleo) y madre categoría } 9 \\
\text { (empleada en empresa de limpieza } \\
\text { industrial) a hijo categoría } 7 \text { (trabajador } \\
\text { de los servicios y comercio de rango } \\
\text { inferior) }\end{array}$ \\
\hline $\begin{array}{l}\text { De padres con nivel de estudios de } \\
\text { formación profesional a hija con nivel de } \\
\text { estudios universitarios }\end{array}$ & $\begin{array}{l}\text { Movilidad } \\
\text { ocupacional } \\
\text { ascendente }\end{array}$ & $\begin{array}{l}\text { De padre con categoría } 4 \text { y madre con } \\
\text { categoría } 7 \text { a hija con categoría } \\
\text { ocupacional } 2\end{array}$ \\
\hline $\begin{array}{l}\text { De padres con nivel de estudios } \\
\text { universitarios a hija con nivel de estudios } \\
\text { universitarios }\end{array}$ & $\begin{array}{l}\text { Movilidad } \\
\text { ocupacional } \\
\text { descendente }\end{array}$ & $\begin{array}{l}\text { De padre y madre con categoría } 4 \text { a } \\
\text { hija con categoría ocupacional } 9\end{array}$ \\
\hline $\begin{array}{l}\text { De padres con nivel de estudios } \\
\text { secundarios a hijo con nivel de estudios } \\
\text { secundarios }\end{array}$ & $\begin{array}{l}\text { Movilidad } \\
\text { ascendente }\end{array}$ & $\begin{array}{l}\text { De padre y madre con categoría } 9 \text { a } \\
\text { hijo con categoría ocupacional } 7\end{array}$ \\
\hline $\begin{array}{l}\text { De padres con nivel de estudios } \\
\text { universitarios a hijo con nivel de estudios } \\
\text { de formación profesional de grado medio }\end{array}$ & $\begin{array}{l}\text { Movilidad } \\
\text { descendente }\end{array}$ & $\begin{array}{l}\text { De padre y madre categoría } \\
\text { ocupacional } 3 \text { a hijo con categoría } \\
\text { ocupacional } 4\end{array}$ \\
\hline
\end{tabular}

Como vemos, una conclusión evidente supondría afirmar que las familias migrantes argentinas tienden a vivenciar una movilidad intergeneracional educativa ascendente tras la migración, teniendo en cuenta que seis de los ocho casos aquí presentados así lo reflejan. Por supuesto, estos resultados no ofrecen la representatividad necesaria para realizar generalizaciones al respecto. Pese a ello, ofrecen algunos indicios del comportamiento de los actores dentro de la estructura social. Ello se traduce en la superación del nivel educativo adquirido por los hijos respecto de aquel con el que cuentan ambos padres.

En el plano ocupacional, por el contrario, encontramos una representación pareja entre los casos que revelan una movilidad ocupacional ascendente de padres a hijos ( 4 casos) yde una movilidad ocupacional descendente (4 casos). 
Estos resultados no irían en la línea de la tesis que sostiene la teoría de la proletarización (Poulantzas, 1973), en la que se prevé una tendencia a la descualificación de los actores sociales, pues el nivel educativo parece no decrecer. Las familias con una movilidad intergeneracional ocupacional descendente confirmarían la tesis marxista y weberiana sobre una sociedad rígida donde la movilidad no siempre sería alcanzable, a pesar del logro educativo. En este fenómeno se refleja el efecto de la sobrecualificación y subocupación juvenil, repetida en casi todos los casos de los jóvenes entrevistados.

Estos resultados también contradecirían la teoría del capital humano, en la que se pone de relieve que la movilidad laboral ascendente está asociada al nivel de estudios (Miguélez, 2011, p. 154). Es decir, a pesar de lograr superar el nivel formativo de padres a hijos, el proceso de inserción al mercado de trabajo revela otros factores que impiden consolidar el logro educativo. Este análisis parece guardar relación con una variable fundamental y que hace referencia al tipo de estructura laboral en Galicia, así como a los procesos de discriminación dentro del mercado de trabajo. Así, una estructura laboral estratificada según origen étnico impediría que los migrantes lograsen el objetivo de la movilidad social de acuerdo al nivel educativo alcanzado y favorecería la consolidación de nichos de empleo etnificados, en los cuales encontrarían un hueco para la inserción ocupacional.

Por otra parte, si observamos las trayectorias ocupaciones de las primeras generaciones, vemos unas tendencias similares entre varones y mujeres en la inserción a la nueva estructura sociolaboral. Ambos presentan una tendencia hacia la reproducción de la categoría ocupacional entre ambos contextos sociales, con una menor representatividad de movilidad ocupacional descendente. No obstante, no existen casos de movilidad ascendente tras la migración. Podríamos decir, a la vista de los resultados, que las primeras generaciones no generan una mejora de las condiciones ocupacionales y que, en todo caso, se sacrifican por el porvenir de los retoños, pues los padres no mejoran su ubicación dentro de la estructura ocupacional, y en ocasiones la empeoran. Los hijos, sin embargo, parecen haber mejorado la potencialidad hacia un mejor puesto de trabajo, pues han adquirido un nivel educativo más elevado, produciéndose una adquisición de capital cultural.

En el próximo apartado presentaremos tres historias familiares que ilustran las cuatro posibilidades estudiadas de movilidad social, desde la medición de las dos dimensiones citadas: la educativa y la ocupacional. Los resultados de este estudio no han mostrado casos de reproducción de la categoría socioprofesional de padres a hijos, pues en el caso de jóvenes insertados/as en el negocio familiar se ha considerado que los descendientes fueron "empleados" dentro del sector de servicios.

\section{Historias familiares de movilidad social intergeneracional}

El primer caso presentado ilustra una movilidad educativa ascendente que supera el nivel de educación secundaria "sin terminar" o "de primer tramo" (equivalente a la ESO en España) de sus padres, con un nivel de estudios de formación profesional y que afortunadamente también se refleja en el movimiento ocupacional ascendente entre generaciones. Se trata del caso de Tomás:

Historia familiar 1: Tomás

Tomás es hijo de Hugo y Delia y el tercero de cuatro hermanos. La familia de Tomás llegó a Galicia de forma escalonada en el año 2002. Primero fue su padre el que desembarcó en Vigo, siguiendo el rumbo de su hermano menor, quien vivía en Galicia desde hacía varias décadas. Hugo es hijo de una gallega emigrada a la Argentina. Sin embargo, nunca había estado antes en la ciudad natal de su madre. Esta apenas le había contado las razones de su migración y Hugo no sabía mucho acerca de sus orígenes gallegos. En Argentina, Hugo trabajaba como chofer de autobús escolar. Su mujer, Delia, era ama de casa. Ambos con un nivel educativo de educación secundaria sin terminar. La familia residía en la localidad de Lomas de Zamora, en el con-urbano bonaerense, zona oeste. A Hugo la idea de la migración ya se le había planteado en varias ocasiones antes, en otros períodos de dificultades económicas y de tensiones vividas a nivel político en el país.

La llegada de Hugo a Galicia se produjo como una estrategia de evaluación sobre las posibilidades de poder encontrar un trabajo para luego reagrupar a su mujer e hijos. La situación en Buenos Aires era crítica para esos años. La familia de Tomás 
pensaba en la migración como opción de mejora de las condiciones de vida. El famoso "corralito" económico del año 2001 había desencadenado una crisis política y social de una importante violencia cívica. Hugo y su mujer temían por la seguridad de sus hijos, quienes habían sido ya asaltados en varias ocasiones cerca de la vivienda familiar. El barrio de residencia del grupo comenzaba a convertirse en un lugar inhóspito. Si bien el "punta de lanza” había sido Hugo, fue sobre todo la motivación del hijo mayor de la fratría, Pedro, la que alentó a todo el grupo a dar el gran paso. El primogénito era el que veía las mayores dificultades en el entorno local dada su edad de entrada al mercado de trabajo local.

Tras algunos meses, Hugo logró insertarse en el mismo sector de actividad en el que trabajaba en su país de origen. En su caso, no hubo un desclasamiento sino un mantenimiento del nivel ocupacional e incluso una mejora del nivel de ingresos a comparación a lo que ganaba en Argentina dentro de la misma categoría ocupacional. Comenzó como chofer de camión en una conocida empresa transportista de la ciudad de Vigo. La estrategia familiar había dado sus frutos. Fue sobre todo Pedro quien se encargó de organizar el periplo del resto de la familia hacia Galicia. Así, fue entre el padre, Delia y el hijo mayor que la migración fue organizada. Al llegar a Galicia Delia se propuso acompañar a sus hijos en el proceso de adaptación al nuevo sistema escolar, si bien tuvo que buscar trabajo rápidamente dada la necesidad económica del grupo. Su ausencia en la casa familiar, sin embargo, tampoco parece haber influenciado el desempeño escolar de sus hijos, si bien reconoce que hubiera querido apoyarles más en sus tareas a la llegada de la escuela.

Los hijos mayores, Pedro y Víctor, fueron los que sin duda tuvieron más dificultades para adaptarse al nuevo sistema educativo. El mayor de los hermanos, Pedro, había recientemente acabado la educación secundaria en Argentina, llegando a Galicia a los 18 años; al llegar realizó un ciclo superior en administración de empresas y enseguida tras terminarlo comenzó a trabajar en un puesto de dependiente en una cadena de comida rápida multinacional. Esta decisión tuvo que ver, en gran parte, con la obligación que familiarmente le era otorgada como fuente de apoyo económico grupal. Víctor, el siguiente de los hermanos, llegó a los 13 años y fue el que más problemas tuvo en la adaptación al nuevo ámbito escolar y en la integración a la nueva sociedad en general. Víctor siempre tuvo en mente la idea de regresar a Argentina. Por esta razón tampoco se planteó una carrera de larga duración en Galicia, teniendo por meta el retorno inminente al acabar con la educación secundaria. Cuando por fin llegó el momento, Víctor viajó a Buenos Aires pero no se encontró del todo cómodo. Regresó a Galicia sin saber bien cómo continuar su trayectoria e ingresó en la empresa donde trabajaba su hermano, como encargado de equipo de la misma multinacional de comida rápida. Fue entonces cuando conoció a su actual esposa y realizó una formación profesional de grado medio de tornero, pero no ha logrado cambiar de sector ocupacional. La posibilidad de realizar carrera universitaria no se planteó como posibilidad en ninguno de los hermanos, tampoco era una exigencia por parte de Hugo y Delia, quienes no transmitían tampoco unas expectativas de estudios superiores en sus hijos.

Tomás tenía diez años cuando llegó a Vigo. Su adaptación en la escuela no fue fácil, cuenta, pero tampoco supuso un proceso traumático como lo fue para su hermano Víctor: la llegada de Víctor en edad adolescente supuso una adaptación mucho más violenta frente al cambio de entorno social. Si bien el aprendizaje del gallego era nuevo para Tomás, la medida de la exención de esta asignatura durante dos años le ayudó a poder pasar de curso mientras aprendía el idioma. Con todo, la adaptación al nuevo entorno escolar produjo un descenso considerable en su nivel de rendimiento escolar, según describe su relato y se confirma en el de su madre. A pesar de no haber tenido nunca problemas con la escuela en Argentina, Tomás comenzaba a tener serias dificultades para aprobar las asignaturas durante su educación secundaria. Vivió además escenas que tanto el como su madre identifican como de "ensañamiento" por parte de algún miembro del profesorado, hecho que vinculan ambos a su origen nacional. Cuando llegó el fin de la etapa de educación obligatoria Tomás ya había repetido el curso en varias ocasiones e intentó continuar con el bachillerato, pero finalmente lo abandonó por su dificultad. La universidad nunca fue una opción para Tomás, ni siquiera la había considerado. La opción que le quedaba era la formación profesional de grado medio o acceder a un ciclo profesional superior mediante una prueba de acceso. Se decantó por una formación superior en mecánica de automoción como primera opción, pero no obtuvo plaza y para no quedarse sin "hacer nada todo el año" optó por comenzar con la formación en diseño de mecánica que había escogido en segundo lugar, la cual abandonó al poco tiempo por el nivel de dificultad y por confirmar que se trataba de una formación que no era francamente de su interés. En el momento de la entrevista, Tomás esperaba su turno para acceder a la formación profesional de grado medio en carpintería, como vía para continuar su trayectoria educativa, pero debía esperar al año siguiente para poder comenzar con el nuevo curso escolar. Mientras tanto, el joven preveía que su hermano mayor le ayudara a conseguir un puesto de dependiente dentro de la cadena multinacional del sector de la hostelería donde este trabajaba.

El segundo perfil que se observa entre los casos estudiados es el de la movilidad educativa descendente y así también lo refleja la posición dentro del mercado de trabajo de Nicolás, si lo comparamos con la categoría socioprofesional de sus padres, produciéndose un descenso dentro de la escala ocupacional. A pesar de contar con posibilidades de acceso a capital económico, social y cultural, Nicolás no logra vencer los obstáculos (estructurales) que desvían su trayectoria hacia un itinerario de baja cualificación. No obstante, a pesar de que Nicolás obtiene una categoría educativa inferior a la de sus padres, se acerca a la categoría ocupacional de estos mediante la transmisión intergeneracional de lo que Thélot (1991) llamaría el "savoir faire", disposición 
y actitud hacia la adquisición de información y de destreza social que le permitieron acceder a un itinerario más ventajoso:

Historia familiar 2: Nicolás

Nicolás nació en Buenos Aires, a los 3 años fue traído por sus padres a vivir a Galicia. Viviana, su madre, es diplomada universitaria en diseño gráfico; su padre comenzó los estudios de periodismo aunque no los terminó. El matrimonio decidió migrar a Galicia a finales de la década de los 90, cuando Argentina mostraba claros indicios de deterioro económico y social tras una larga época de política neoliberal. Diego, el padre de Nicolás, ya había estado varias veces antes en Galicia, antes de contraer matrimonio con Viviana, e incluso había probado la experiencia de quedarse por un tiempo a trabajar en la ciudad de Vigo, de donde eran sus padres y donde tenía gran cantidad de familiares y amigos.

La migración a Galicia para esta familia no era una historia nueva, si bien ni Viviana ni Nicolás habían estado nunca en esta parte del Estado español.

Diego fue el primero que viajó en este último proyecto de movilidad geográfica. En Vigo tenía redes familiares, pero sobre todo de conocidos y amigos que le facilitaron el acceso a un puesto de trabajo. Esta vez comenzó empleado como camarero. Mientras tanto Viviana preparaba todo para viajar también junto a su marido, trayendo a Nicolás, su único hijo hasta el momento.

Diego enseguida comenzó a vincularse con redes relacionadas al ámbito de la política, particularmente a un sindicato que tenía vínculos estrechos con el sector sindicalista en Buenos Aires. Esta fue su principal fuente de capital social y que permitió su inserción en la actividad política de la ciudad de Vigo. Su mujer también consiguió un puesto de trabajo gracias a estos contactos, como mediadora intercultural en la sede de la institución sindicalista situada en dicha localidad.

La incorporación de Nicolás al sistema escolar gallego se produjo sin problemas pues el menor llegó en un momento previo a la escolarización, lo que supuso que su trayectoria educativa fuera íntegramente desarrollada en Galicia. Su paso por la escuela, sin embargo, comenzó a ser bastante conflictivo llegada la edad de la adolescencia, presentando algunas dificultades en sus estudios y una ausencia de motivación para acabar con la etapa de educación secundaria. Ello explica su poca voluntad de continuar con estudios superiores y su decisión de querer orientarse hacia una formación de tipo profesional, una vez terminada la ESO. A pesar de la insistencia de sus padres, profesionales ambos con nivel de educación terciaria y superior, Nicolás no quiso continuar con el bachillerato y optó por la carrera más corta. Sin embargo, a partir de la culminación de sus estudios en la etapa escolar, Nicolás ha venido encadenando diferentes formaciones profesionales sin saber bien qué camino tomar, al no encontrar una orientación clara para insertarse en el mercado de trabajo local. Su última opción se orienta hacia una formación profesional de grado superior en el área de deportes. Con todo, Nicolás ha comenzado a realizar trabajos de forma independiente como profesional de las artes gráficas, gracias a su titulación de formación profesional en diseño gráfico.

El caso de la familia de Nicolás es además un buen ejemplo para observar la movilidad espacial circular entre generaciones de migrantes que vinculan Buenos Aires con Galicia. Es también reflejo de un proceso de transmisión del capital humano familiar y revela cómo este contribuye a orientar las trayectorias ocupacionales- de los descendientes. La inestabilidad en el empleo, como fenómeno que se extiende a la totalidad de la población joven en Galicia, lleva a Nicolás a buscar un camino que permita la entrada a un puesto de trabajo dentro de la administración pública.

Volvemos a insistir sobre el hecho de que si bien el nivel educativo de los retoños ha superado el de sus padres, ello no supuso una mejora en la posición adquirida en el mercado laboral teniendo en cuenta los factores estructurales del momento (crisis del mercado de trabajo, empleo juvenil precario, sobrecualificación y subocupación juvenil).

Un último caso, el de Marta, nos permitirá observar estas barreras macrosociológicas de forma más clara, restricciones que explican este tipo de trayectorias de movilidad social "truncadas":

Historia familiar 3: Marta

Marta llegó a Galicia cuando tenía 9 años. Su madre fue la que migró primero. Llegó a Galicia buscando nuevas oportunidades laborales. En Argentina se dedicaba al arte plástica y la falta de recursos e incentivos en este sector hicieron que se planteara la idea, junto a su compañero sentimental, de una migración al extranjero conducente a mejorar las condiciones de vida. Mirta, la madre de Marta, contaba con redes familiares en la provincia de Lugo. Allí desembarcaron como primer lugar de residencia Mirta, dos de sus hijas y su pareja del momento, padre de una de las jóvenes. En Argentina había quedado la hermana mayor de la fratría, resultado de un matrimonio anterior. La separación de su hija, cuenta Mirta, ha sido una de las peores dificultades a afrontar en la migración. Mirta había logrado insertarse en el sector de la artesanía al llegar a Galicia. Consiguió, a partir 
de su trabajo, ganar un reconocimiento dentro del sector artesanal gallego, pero la naturaleza de su profesión no le permitía acceder a una estabilidad económica.

Tanto la madre como el padre de Marta cuentan con estudios universitarios en el ámbito de las artes plásticas. Marta recuerda que su integración en la escuela fue difícil por la dificultad añadida del aprendizaje de la lengua gallega y por el desajuste del calendario escolar entre su país de origen y el sistema educativo local, lo que hizo que tuviera que adaptarse al curso acorde a su edad pero en una etapa muy avanzada. Más tarde, fue afrontando dificultades en los últimos cursos de la educación primaria, repitiendo en $8 .^{\circ}$ de EGB. Marta logró terminar la etapa de enseñanza secundaria obligatoria, obtuvo su título de bachillerato y no dudó en acceder a la universidad. La joven se graduaría en Bellas Artes, añadiendo a ello un postgrado en psicopedagogía. Al terminar con sus estudios, Marta no encontró una vía de inserción fácil al mercado de trabajo local. Cuenta la joven que la situación familiar era de bastante inestabilidad. Marta tuvo que afrontar situaciones de mucha dificultad para conseguir acabar la carrera universitaria. Por esta razón, Marta comenzó a trabajar en la época estival cuando tenía 17 años, bajo la autorización de su madre. Su primer empleo fue como personal de limpieza en un hotel. Frente a la imposibilidad de compaginar el horario lectivo con una jornada laboral, intentó ganar algo de dinero en ilustraciones de libros para editoriales, pero tampoco lograba solucionar la situación de precariedad. Al concluir con la especialización, Marta intentó nuevamente encontrar un puesto de trabajo acorde a su formación, pero no tuvo suerte. La joven buscó rápidamente una fuente de ingresos en otros sectores de actividad. Dada su experiencia laboral anterior, Marta sigue trabajando como empleada doméstica en una vivienda de familia. Marta no ha logrado insertarse profesionalmente en su campo de estudio ni cumplir con sus expectativas profesionales aún. La joven cree que su falta de suerte se debe en gran parte al contexto en que reside, donde no existen muchas posibilidades de inserción laboral para la población joven y menos dentro de su ámbito de trabajo. Sin embargo, Marta tampoco parece estar del todo dispuesta a emprender una nueva movilidad geográfica hacia regiones de España que ofrezcan mejores posibilidades. Cuando reflexiona sobre las posibilidades de empleo en su país de origen, que observa por la experiencia de amigas y por la de su hermana mayor que aún reside en Argentina, la joven concluye que el entorno de donde proviene sea finalmente un mejor contexto para el empleo joven, especialmente para aquellos que obtienen una titulación universitaria. En sus planes, al menos por el momento, no existe la posibilidad de un retorno inmediato, pues remarca que su vínculo sentimental actúa como elemento de anclaje de su vida en Galicia.

El caso de Marta revela cómo los jóvenes protagonistas de una migración anterior se ven limitados en sus nuevas y futuras elecciones de movilidad geográfica y por ende, en su movilidad ocupacional, pues limitan también las posibilidades de empleo a un ámbito territorial concreto con pocas oportunidades laborales. Entendemos que esto se produce como resultado de una experiencia migratoria familiar dolorosa que parece haber dejado huella. Vemos, a partir de ello, cómo las limitaciones a nivel emocional influyen en unas trayectorias laborales, pues estos, si bien no han renunciado a sus planes en el ámbito educativo, se encuentran inmersos en un contexto social no elegido y que ofrece pocas alternativas de inserción profesional.

\section{Conclusiones:}

Si volvemos la mirada hacia el marco teórico escogido para este trabajo, la teoría de Portes y Böröcz (1989) parece contribuir a comprender cómo las posiciones sociales de estas familias parecen situarse dentro de un modelo más aventajado que los nichos de empleo más tradicionales de la población migrante en esta región, generalmente situados dentro de la economía sumergida. Los procesos de incorporación al mercado de trabajo se producen, así, en el marco del mercado primario. Podemos decir que en el caso de nuestros informantes, los migrantes han ocupado puestos que, en términos de la clasificación que realizan estos dos autores, describirían en general al pequeño empresariado e incluso a ocupaciones técnicas dentro de las llamadas posiciones de "liderazgo profesional y cívico".

Ya otros trabajos han concluido que los diferentes espacios sociales, el de origen y el de destino, "se entreteje desde la conceptualización de las circulaciones migratorias, entendidas como un recurso para la reproducción social de los trabajadores y trabajadoras, de sus familias y de sus comunidades" (Jiménez Zunino, 2018). Las conclusiones que se desprenden de las historias analizadas nos llevan a reflexionar sobre estos procesos de reproducción familiar desde el prisma de la movilidad educativa y de la movilidad ocupacional. Comenzaremos por la dimensión educativa de la movilidad social para seguir con las conclusiones sobre la categoría socioprofesional. 
En primer lugar, y acerca de nuestra pregunta sobre si el resultado de la posición social alcanzada por los padres en el momento posterior a la migración contribuyó a obstaculizar o a facilitar las trayectorias de movilidad social de los hijos, podemos decir con toda seguridad que no supusieron una fuente de identificación para la mejora de la posición social de estos últimos, pues en ningún caso se produjo una mejora de la categoría socioprofesional alcanzada en Argentina.

La movilidad educativa ascendente tiene una triple explicación. Por una parte, la falta de empleo joven que llevó a los retoños a sobre formarse y encadenar cualificaciones frente a la falta de oportunidades. En segundo lugar, no debe olvidarse el importante esfuerzo realizado en España desde la década de los 80 para integrar a los jóvenes al sistema educativo con la generalización de los estudios postobligatorios (Echeverría Zabalza, 1998, p. 378). En tercer lugar, nuestra interpretación de las aspiraciones escolares de los hijos de inmigrantes no se basa en el resultado de la estrategia de movilidad social a partir de la movilidad espacial de las primeras generaciones, sino que se orienta, en línea con otros trabajos desarrollados en la materia (Ichou, 2018) y en otros contextos sociales, con el lugar que ocupa el valor educativo en los proyectos migratorios de estas familias, sea como aspiración a la mejora de las condiciones de vida, sea como recurso familiar basado en un capital cultural que define las disposiciones y actitud hacia el aprendizaje. Para ello, no será la categoría ocupacional alcanzada sino el nivel de estudios de los padres lo que, según la teoría bourdiana y de la transmisión del capital cultural, nos aportaría más indicios para extraer conclusiones acerca de las motivaciones que condujeron a estos jóvenes a realizar una u otra elección educativa.

De esta forma, las tres teorías sobre estratificación social revisadas en el apartado teórico (teoría de la industrialización, de la proletarización y del capital humano) se aúnan de esta forma en dos conclusiones fundamentales que explican los movimientos observados. Por un lado, la importantísima influencia del capital cultural familiar y de su transmisión entre las generaciones en forma de disposición frente a la educación, hacia la obtención de diplomas: lo vemos en el caso de familias con padres con estudios universitarios en las que se reproduce este nivel en sus hijos y, de la misma forma, en las familias con niveles de estudios secundarios con hijos que no superan el nivel de formación profesional. Y por el otro lado, el decisivo impacto de los factores estructurales y coyunturales de la sociedad de acogida en un momento dado, pues vemos que si bien los jóvenes migrantes alcanzan niveles de estudios universitarios, estos no se corresponden con las categorías socioprofesionales alcanzadas. El resultado del balance entre el peso de ambos determinantes sociológicos se corresponderá entonces con la calidad de los recursos disponibles y con la forma en la que los mismos son utilizados, definido en lo que Zhou y Lin (2005) llamaron el "capital étnico"; en definitiva: la intersección de los diferentes capitales familiares (social, económico, cultural).

\section{REFERENCIAS}

Actis, W. y Esteban, F. (2007). Argentinos hacia España (« sudacas » en tierras « gallegas »): el estado de la cuestión. En S. Novick (coord.), Sur-Norte: estudios sobre la emigración reciente de argentinos. Universidad de Buenos Aires: Editorial Catálogos.

Aparicio, R. (2007b). Las "segundas generaciones" en España:¿Qué movilidad social. En J. Igartua, y C. Muñiz, Medios de comunicación, Inmigración y Sociedad (pp. 311-341). Salamanca, Ed. Univesidad de Salamanca.

Blalock, H.M. (1967). Toward a theory of minority-group relations. New York: Wiley.

Bonacich, E. (1973). A theory of middleman minorities. American sociological review, 38(5), 583-594.

Bourdieu, P. (1979). Los Tres Estados del Capital Cultural. Sociológica, 5, 11-17.

Bourdieu, P. y Passeron, J.C. (1970). La Reproduction: Éléments d'une théorie du système d'enseignement. Paris: Les Editions de minuit.

Bowles, S. y Gintis, H. (1976). Schooling in capitalist America. New York: Basic Books.

De Singly, F. y Thélot, C. (1986). Racines et Profils des Ouvriers et des Cadres Supérieurs. Revue française de sociologie, $27,47-86$ 
Echeverría Zabalza, J. (1998). Movilidad social y comportamiento electoral. Reis, Revista española de investigaciones sociológicas, 237-267.

Echeverría Zabalza, J. (1999). La movilidad social en España, 1940-1991. Madrid: Ediciones AKAL.

Feito Alonso, R. (2010). Escuela y democracia. Política y Sociedad, 47(2), 47-61.

Goldthorpe, J.H. y Hope, K. (1974). The social grading of occupations: A new approach and scale. Oxford: Clarendon Press.

Ichou, M. (2015). Migration background and educational inequality: a longitudinal study of the academic achievement of children of immigrants in France and England. Revue française de pédagogie, (2), 29-46.

Ichou, M. (2018). Les enfants d'immigrés à l'école. Inégalités scolaires, du primaire à l'enseignement supérieur. Collection Education et société. Paris: Editions PUF.

Jiménez Zunino, C. (2018). Mercados de trabajo: algunas lecturas renovadas sobre las migraciones laborales. Temas de Antropología y Migración, 10. Instituto de Lingüística. Facultad de Filosofía y Letras. Universidad de Buenos Aires. Disponible en el Repositorio Digital Institucional de la Universidad de Buenos Aires: http://www.migra ntropologia.com.ar/images/stories/PDF/Revista10/parte1.pdf

Laiz, S. (2016) "Familias migrantes y estrategias de movilidad social intergeneracional en las migraciones argentinas y marroquíes a Galicia : un enfoque inter-seccional" en "Procesos migratorios y dinámicas familiares desde una perspectiva de género. Circuitos en, desde y hacia América Latina” (Coords.) Gil Araujo, S. et Rosas, C. Odisea. Revista de Estudios Migratorios, (3) 78-98. Ed. Instituto de Investigación Gino Germani, Univerdad de Buenos Aires, Argentina.

Meurs, D. y Pailhé, A. (2008). Descendantes d'immigrés en France: une double vulnérabilité sur le marché du travail? Travail, genre et sociétés, 2, 87-107.

Michelat, G. y Simon, M. (1977). Classe, religion et comportement politique. Paris: Editions sociales.

Miguélez, F. (2011). Trayectorias laborales de los inmigrantes en España. Quit, Centre d'Estudis Sociòlogics sobre la Vida Quotidiana i el Treball. Barcelona: Universitat Autònoma de Barcelona.

Pedreño Cánovas, A. (coord.) (2013). "Que no sean como nosotros". Trayectorias formativo-laborales de los hijos de familias inmigrantes en el campo murciano. Murcia: EDITUM publicaciones de la Universidad de Murcia.

Percheron, A. (1991). La transmission des valeurs. En F. De Singly (ed.), La famille. L'état des savoirs (pp. 183-193). Paris: La découverte.

Portes, A. y Böröcz, J. (1989). Contemporary immigration: theoretical perspectives on its determinants and modes of incorporation. International Migration Review, special Silver Anniversary Issue: International Migration an Assessment for the 90's, 23(3), 606-630.

Poulantzas, N. (1973). Las clases sociales en América Latina. Méjico: Ed. Siglo XXI.

Requena, M., Salazar, L. y Radl, J. (2012). Estratificación social. Madrid: McGraw Hill.

Rumbaut, R. G. (2004). Ages, life stages, and generational cohorts: decomposing the immigrant first and second generations in the United States 1. International migration review, 38(3), 1160-1205.

Sánchez Hugalde, A. (2004). Movilidad intergeneracional de ingresos y educativa en España (1980-90). En XI Encuentro de Economía Pública: los retos de la descentralización fiscal ante la globalización. Barcelona: Encuentro de Economía Pública.

Thélot, C. (1991). La mobilité sociale. La Famille. L'État des Savoirs. Paris: La Découverte.

Zhou, M. \& Lin, M. (2005). Community transformation and the formation of ethnic capital: Immigrant Chinese communities in the United States. Journal of Chinese overseas, 1(2), 260-284.

\section{Notas}

1 Crisis financiera que tuvo como consecuencia la "congelación" de los ahorros privados y una profunda crisis políticosocial. 
2 Definidas por Rumbaut (2004) como las generaciones de hijos de migrantes llegadas al país de inmigración a la edad escolar.

3 El sistema ESeC fue teorizado por Goldthorpe, desde la corriente neo-weberiana, y presenta una categorización que agrupa aquellas ocupaciones codificadas con tres dígitos de la CIUO-88, logrando mantener las categorías en una posición similar dentro del mercado de trabajo y, al mismo tiempo, permitiendo otras agrupaciones más sintéticas que reducen la complejidad de la clasificación (Informe España CECS, 2011, p. 309).

4 Los tres elementos que definen el origen social, tomando como base la definición realizada por Bourdieu y Passeron (1970), serían los siguientes: el nivel educativo de los padres; la categoría ocupacional y el nivel socioeconómico de los padres; el origen social rural o urbano de procedencia.

5 Nos hemos encontrado con múltiples trayectorias educativas aún no acabadas o prolongadas en su paso a la inserción laboral frente a la situación de falta de empleo estructural. 